Un médiévalisme scolaire : le traitement de la période médiévale dans les manuels scolaires de classe de seconde, édition 2019

Florian Besson

\author{
(2) OpenEdition \\ Journals \\ Édition électronique \\ URL : https://journals.openedition.org/cem/18574 \\ DOI : $10.4000 /$ cem. 18574 \\ ISSN : 1954-3093 \\ Éditeur \\ Centre d'études médiévales Saint-Germain d'Auxerre
}

Référence électronique

Florian Besson, « Un médiévalisme scolaire : le traitement de la période médiévale dans les manuels scolaires de classe de seconde, édition 2019 », Bulletin du centre d'études médiévales d'Auxerre I

BUCEMA [En ligne], 25.2 | 2021, mis en ligne le 31 décembre 2021, consulté le 22 septembre 2022

URL : http://journals.openedition.org/cem/18574; DOI : https://doi.org/10.4000/cem.18574

Ce document a été généré automatiquement le 22 septembre 2022.

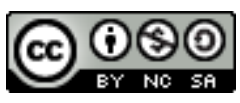

Creative Commons - Attribution - Pas d'Utilisation Commerciale - Partage dans les Mêmes Conditions 4.0 International - CC BY-NC-SA 4.0

https://creativecommons.org/licenses/by-nc-sa/4.0/ 


\section{Un médiévalisme scolaire : le traitement de la période médiévale dans les manuels scolaires de classe de seconde, édition 2019}

\section{Florian Besson}

Les nouveaux programmes d'histoire du secondaire, entrés en vigueur en septembre 2019 dans le cadre d'une réforme de grande ampleur du lycée, prescrivent de consacrer un chapitre à l'histoire médiévale en classe de seconde générale, sur huit chapitres en tout. Il s'agit du chapitre deux, intitulé «La Méditerranée médiévale: espace d'échanges et de conflits à la croisée de

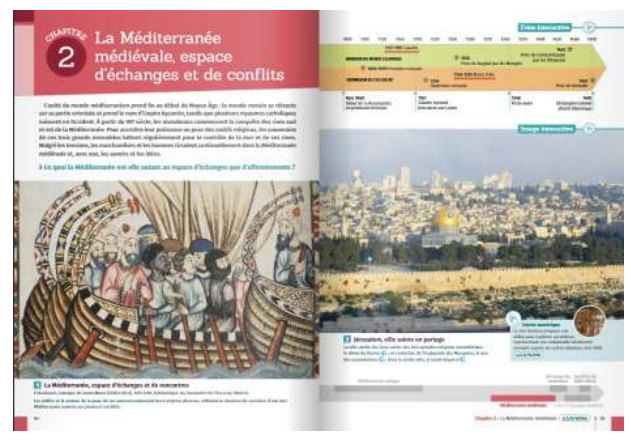
trois civilisations ». Il fait suite à un chapitre introductif consacré à la notion de périodisation - l'une des nouveautés de ces programmes - et à un premier chapitre portant sur l'Antiquité grecque et romaine. Le Bulletin officiel présente le chapitre ainsi (fig. 1) : 
Fig. 1. Légende. Extrait du Bulletin officiel spécial $n^{\circ} 1$ du 22 janvier 2019, fixant le programme d'histoire-géographie de seconde générale et technologique.

Chapitre 2. La Méditerranée médiévale : espace d'échanges et de conflits à la croisée de trois civilisations

\begin{tabular}{|l|l|}
\hline Objectifs du chapitre & $\begin{array}{l}\text { Ce chapitre vise à montrer comment des civilisations entrent en } \\
\text { contact, nouent des relations et connaissent des conflits dans un } \\
\text { espace marqué par les monothéismes juif, chrétien et musulman. } \\
\text { On peut mettre en avant : } \\
\text { - l'émergence de grands ensembles de civilisation; } \\
\text { - les contacts et les heurts entre Chrétienté et Islam; } \\
\text { - l'hétérogénéité religieuse et politique entre Rome et Byzance et au } \\
\text { sein du monde musulman; } \\
\text { - la persistance de la circulation de biens, d'hommes et d'idées } \\
\text { dans cet espace méditerranéen relié à l'Europe du Nord, à l'Asie } \\
\text { et l'Afrique. }\end{array}$ \\
\hline $\begin{array}{l}\text { Points de passage et } \\
\text { d'ouverture }\end{array}$ & $\begin{array}{l}\text { - Bernard de Clairvaux et la deuxième croisade. } \\
\text { - Venise, grande puissance maritime et commerciale. }\end{array}$ \\
\hline
\end{tabular}

Il ne s'agira pas ici de commenter les choix pédagogiques et scientifiques qui soustendent ce programme, ce qui a déjà été fait par d'autres, souvent sur le mode de la critique sévère ${ }^{1}$. Tout au plus peut-on rapidement rappeler combien l'intitulé de ce chapitre, ainsi que les différents objectifs détaillés par le Bulletin officiel, sont finalement très classiques. Les programmes de 1995, en effet, imposaient déjà un chapitre sur le Moyen Âge en classe de seconde, intitulé «La Méditerranée au XII siècle (1095-1204) : carrefour de trois civilisations ». Certes, l'ampleur chronologique était alors bien moindre : à peine plus d'un siècle, de la première à la quatrième croisade, quand le programme actuel impose d'embrasser une période floue, allant au moins du début du $\mathrm{XI}^{\mathrm{e}}$ siècle à la fin du $\mathrm{XV}^{\mathrm{e}}$ siècle; le tout en moins d'heures qu'en 1995, ce qui nourrit évidemment une série de critiques émanant des historien-nes et des institutions savantes comme la Société des historiens médiévistes de l'enseignement supérieur public (SHMESP). Reste que ce nouveau chapitre ressemble de près à celui de 1995, rompant donc avec l'expérience tentée par les programmes de 2010 qui se recentraient sur l'Occident chrétien de l'époque féodale, avec au choix un chapitre sur les villes ou sur les campagnes. Pour classique, voire convenu qu'il soit, ce chapitre ne s'en inscrit pas moins dans une actualité historiographique évidente, bien visible à travers le grand nombre de thèses, d'ouvrages ou d'expositions qui portent dernièrement sur la Méditerranée médiévale en général et en particulier sur les contacts - économiques, culturels, militaires, intellectuels... - entre ce que le programme appelle «des civilisations ». Ce champ de recherche, dont la vitalité est sans nul doute alimentée par le fait qu'il semble recouper un certain nombre de débats politiques et sociaux contemporains, notamment sur la place de l'islam dans la société française, se cristallise également dans plusieurs questions inscrites ces dernières années au programme des concours de recrutement des enseignants d'histoire-géographie. Ainsi est-il apparu à l'agrégation externe en 2000 sous l'intitulé « Relations des pays d'Islam avec le monde latin du milieu du $\mathrm{x}^{\mathrm{e}}$ au milieu du XIII ${ }^{\mathrm{e}}$ siècle» puis sous la forme «Gouverner en terre d'Islam ( $\mathrm{x}^{\mathrm{e}}-\mathrm{XV}^{\mathrm{e}}$ siècle) » en 2014, et à l'agrégation externe d'histoire en 2019-2020 sous l'intitulé «Juifs, chrétiens et musulmans en Méditerranée, $\mathrm{XI}^{\mathrm{e}}-\mathrm{XV}^{\mathrm{e}}$ siècle ». Il y a donc, de toute évidence, une actualité - à la fois scientifique, pédagogique et sociale - de la Méditerranée médiévale, qui nourrit largement l'architecture de ces nouveaux programmes du secondaire. 
3 Loin donc de vouloir analyser les arrière-plans pédagogiques, politiques voire idéologiques de ce programme, le présent article s'intéressera plutôt à l'une de ses émanations les plus tangibles, à savoir les manuels scolaires. À chaque réforme des programmes, plusieurs éditeurs s'emploient en effet à publier - souvent dans l'urgence, ce qui est particulièrement vrai dans le cas de cette réforme-ci - de nouveaux manuels, conformes aux «nouveaux programmes ». Ces supports, diffusés à des centaines de milliers d'exemplaires à travers tout le territoire français - et même au-delà, du fait du réseau des lycées français à l'étranger -, jouent un rôle essentiel tant dans la diffusion des connaissances auprès des élèves que dans l'appropriation de ces nouveaux programmes par les enseignant-es. L'impact effectif de ces manuels scolaires ne doit pas être surestimé : outre la question, difficile à cerner, de leur utilisation réelle par les élèves, rappelons évidemment qu'un manuel est toujours complété par un cours, construit par des professeures qui ne manquent jamais de croiser les manuels, ni de les compléter par d'autres ressources - livres d'universitaires, revues spécialisées comme L'Histoire, musées, sites Internet, podcasts, romans, vidéos YouTube, etc. Même sans tenir compte du fait qu'un manuel n'est jamais qu'un fragment muet d'un cours toujours autrement plus riche, il est largement permis de penser que les élèves euxmêmes ne reçoivent pas passivement les images et les textes de leurs manuels: on connaît notamment le goût du public jeune pour des vidéos YouTube de « vulgarisation historique ", dont certaines sont aujourd'hui d'une qualité et d'une inventivité qui n'a rien à envier à bien des travaux universitaires ${ }^{2}$. Pour le dire plus simplement, le rapport qu'entretiennent les lycéen.nes avec le Moyen Âge ne se construit pas uniquement, tant s'en faut, à travers la poignée de pages que lui consacrent leurs manuels scolaires, de même que les savoirs des élèves ne sont pas uniquement façonnés par les cours ${ }^{3}$.

Il n'en demeure pas moins que ce chapitre d'histoire médiévale constitue le seul contact scolaire qu'un'e lycéen-ne a avec le Moyen Âge entre la classe de cinquième et la fin du lycée. Pour l'écrasante majorité des lycéen-nes qui ne poursuivent pas, après avoir obtenu leur baccalauréat, d'études d'histoire, ce chapitre peut même constituer le dernier contact scolaire avec la période médiévale. Il semble dès lors pertinent d'analyser ces manuels, pour se demander comment ils traitent ce chapitre d'histoire médiévale, comment ils l'illustrent, quels exemples ils exploitent, quels acquis historiographiques ils mobilisent. L'enjeu n'est rien moins que la construction de ce qu'on pourrait appeler un "médiévalisme scolaire", autrement dit une certaine représentation de la période médiévale, forgée par les manuels scolaires et diffusée ainsi à un public lycéen ${ }^{4}$.

\section{Corpus et méthode}

5 En septembre 2019, sept manuels d'histoire différents s'offraient au choix des professeur-es : un manuel édité par Belin, dirigé par David Colon ; deux manuels édités par Nathan, dirigés respectivement par Sébastien Cote et Guillaume Le Quintrec ; un manuel édité par Magnard, dirigé par Jean-Marc Vidal ${ }^{5}$; un manuel édité par Hachette, dirigé par Michaël Navarro et Henri Somonneau ${ }^{6}$; un manuel édité par Hatier, dirigé par Martin Ivernel ; et enfin un manuel édité par LeLivreScolaire.fr, dirigé par l'auteur de cet article ${ }^{7}$. À noter qu'il n'est jamais possible de connaître le ou les auteur'es de chaque chapitre, ces derniers étant présentés collectivement sur la page de garde. On se référera donc dans les pages qui suivent aux manuels comme à des ouvrages 
collectifs, ce qui offre également l'avantage de ne pas pointer du doigt une personne lorsqu'on analysera un certain nombre d'erreurs ou de raccourcis.

6 La position de directeur d'ouvrage que j'ai occupée durant deux ans m'a permis de mieux saisir les mécanismes complexes qui président à la création de manuels scolaires. Ceux-ci, en effet, font intervenir à des moments différents des acteurs distincts, qui n'ont pas tous les mêmes connaissances, les mêmes attentes ni même forcément la même vision de ce que doit être un manuel scolaire. Auteur ou autrice du chapitre - le plus souvent des professeures du secondaire, parfois des professeures de classe préparatoire aux grandes écoles, plus rarement des universitaires -, comité scientifique composé d'universitaires spécialistes de la question mais rarement au fait des programmes, personnel de l'inspection générale particulièrement concerné par les enjeux didactiques, cartographes, graphistes : tous et toutes contribuent à faire d'un manuel scolaire ce qu'il est. Avant de se pencher plus précisément sur une analyse comparative des contenus, il est important de rappeler que le processus de rédaction d'un manuel scolaire est particulièrement contraint. Il l'est d'abord, évidemment, par les bornes assez strictes que fixent les programmes officiels : pour l'histoire médiévale, par exemple, deux "Points de passage et d'ouverture ", respectivement consacrés à "Bernard de Clairvaux et la deuxième croisade » et à "Venise, puissance maritime et commerciale » sont ainsi imposés et se retrouveront donc dans tous les manuels. Il l'est ensuite temporellement : ayant eu la version définitive des programmes durant le mois d'octobre 2018, les éditeurs ont dû boucler une version définitive des manuels fin avril 2019 au plus tard, pour que des spécimens papiers puissent être distribués aux professeures avant la fin de l'année scolaire. En à peine plus de six mois, il a donc fallu faire rédiger le chapitre - ce qui suppose, pour son auteur.rice, de se mettre à jour historiographiquement, de trouver les documents, d'écrire les cours -, le relire, le faire relire, au fil de plusieurs versions successives, mais également faire réaliser les cartes, trouver les images, les faire retoucher, etc. Il s'agit également d'un processus très contraint économiquement: si les textes sont tous libres de droits pour la période médiévale, ce n'est pas le cas de l'iconographie, dont les droits d'usage peuvent souvent être onéreux. D'où la tendance des manuels à utiliser massivement des illustrations tirées de "banques d'images", des banques de données en ligne, comme AKG, Alamy, Bridgeman, etc., qui permettent de trouver rapidement des images à prix réduit. Les enjeux économiques étant élevés - un manuel se vend en moyenne à 25 euros et il s'en vend plusieurs centaines de milliers d'exemplaires -, les relations entre les différentes maisons d'édition sont de l'ordre de la concurrence : sauf exception, les auteur'es ou les directeurs d'ouvrages n'échangent pas les un.es avec les autres. L'objectif premier des maisons d'édition, sinon forcément des directeurs de collection eux-mêmes, est de vendre le plus d'exemplaires possible: la maquette et les contenus sont donc pensés avant tout pour séduire les enseignantes, mais aussi pour plaire aux élèves. Enfin, le processus est contraint par la nature même de l'objet produit : il faut en effet rédiger un manuel synthétique, adapté au volume horaire assez réduit dévolu à chaque chapitre - en l'occurrence, si l'on suit les instructions officielles, cinq ou six heures. Le nombre de pages d'un chapitre oscille ainsi entre 26 et 34 , et il va de soi que vouloir « tout dire » dans un espace aussi réduit tient moins de la gageure que de l'impossible (Tab. 1) $)^{8}$ 
Tab. 1. Nombre de pages du chapitre d'histoire médiévale dans les différents manuels.

\begin{tabular}{|l|l|l|l|}
\hline & $\begin{array}{l}\text { Nombre de pages du chapitre } \\
\text { d'histoire médiévale }\end{array}$ & $\begin{array}{l}\text { Nombre de pages total } \\
\text { du manuel }\end{array}$ & Ratio \\
\hline Belin Colon & 34 & 304 & $11,1 \%$ \\
\hline Hachette & 28 & 288 & $9,7 \%$ \\
\hline Hatier Ivernel & 32 & 304 & $10,5 \%$ \\
\hline $\begin{array}{l}\text { LeLivreScolaire } \\
\text { Besson }\end{array}$ & 26 & 336 & $7,7 \%$ \\
\hline Magnard Vidal & 32 & 288 & $11,1 \%$ \\
\hline Nathan Cote & 26 & 288 & $9 \%$ \\
\hline Nathan Le Quintrec & 26 & 288 & $9 \%$ \\
\hline
\end{tabular}

7 Graphiquement, et malgré des variations de maquettes, tous ces manuels se ressemblent beaucoup, correspondant à la nouvelle ligne éditoriale qui s'est imposée entre 1993 et $1995^{\circ}$. Des doubles pages de cours, entre deux et quatre par chapitre, alternent avec des doubles pages proposant entre trois et sept documents liés à un sujet précis, intitulées généralement « Dossiers », « Documents » ou "Études », double pages dont le nombre est assez variable, puisqu'il oscille entre 4 et 8 (Tab. 2).

Tab. 2. Les « Dossiers » supplémentaires - sans compter les deux «Points de passage et d'ouverture » imposés - dans les différents manuels.

\begin{tabular}{|l|l|l|}
\hline & $\begin{array}{l}\text { Nombre de pages } \\
\text { documentaires } \\
\text { supplémentaires }\end{array}$ & Sujets de ces pages \\
\hline Belin Colon & 6 & $\begin{array}{l}\text { Palerme, une capitale à la croisée de trois } \\
\text { civilisations } \\
\text { Tolède, un centre majeur de traductions } \\
\text { Las Navas de Tolosa : vers une Méditerranée } \\
\text { latine } \\
\text { La cathédrale de Monreale en Sicile } \\
\text { [Empreintes de l'histoire] L'ordre des Templiers } \\
\text { dans Cross Fire } \\
\text { [Empreintes de l'histoire] Cordoue, ville } \\
\text { espagnole marquée par l'héritage d'Al-Andalus }\end{array}$ \\
\hline
\end{tabular}




\begin{tabular}{|c|c|c|}
\hline Hachette & 5 & $\begin{array}{l}\text { La péninsule Ibérique, marquée par trois grandes } \\
\text { civilisations } \\
\text { Contacts et heurts entre chrétienté et islam en } \\
\text { Terre sainte } \\
\text { Palerme } \\
\text { Tolède, un carrefour de culture } \\
\text { Le Destin de Youssef Chahine }\end{array}$ \\
\hline Hatier Ivernel & 8 & $\begin{array}{l}\text { Trois grands ensembles de civilisations } \\
\text { L'Occident chrétien } \\
\text { Byzance et la chrétienté orthodoxe } \\
\text { Le monde arabo-musulman } \\
\text { Croisades et jihad } \\
\text { La Méditerranée, un carrefour du commerce } \\
\text { Le côtoiement religieux } \\
\text { Les échanges culturels }\end{array}$ \\
\hline $\begin{array}{l}\text { LeLivreScolaire } \\
\text { Besson }\end{array}$ & 4 & $\begin{array}{l}\text { Les Templiers, intermédiaires entre Orient et } \\
\text { Occident } \\
\text { Le sucre égyptien, un itinéraire méditerranéen } \\
\text { Palerme, capitale multiculturelle } \\
\text { [Atelier de Clio] Les apports de l'archéologie }\end{array}$ \\
\hline Magnard Vidal & 5 & $\begin{array}{l}\text { Constantinople, capitale byzantine } \\
\text { La Méditerranée des trois monothéismes } \\
\text { La traduction du Coran } \\
\text { Palerme au XII siècle, un creuset culturel } \\
\text { [Regard critique] La croisade, choc ou } \\
\text { rencontre? }\end{array}$ \\
\hline Nathan Cote & 4 & $\begin{array}{l}\text { Le monde musulman, religion et civilisation } \\
\text { L'Empire byzantin et l'Occident } \\
\text { [De la source à l'histoire] Comment les historiens } \\
\text { expliquent-ils le patrimoine archéologique et } \\
\text { monumental de la ville de Jérusalem? } \\
\text { [Passé présent] Pourquoi peut-on dire que la } \\
\text { Méditerranée est un espace majeur de } \\
\text { migrations? }\end{array}$ \\
\hline $\begin{array}{l}\text { Nathan } \\
\text { Quintrec }\end{array}$ & 4 & $\begin{array}{l}\text { La Méditerranée, une zone d'affrontements } \\
\text { violents } \\
\text { La Méditerranée, un espace commercial majeur } \\
\text { Les juifs, une minorité dans l'Espagne chrétienne } \\
\text { La Sicile, un carrefour entre les civilisations de } \\
\text { Méditerranée }\end{array}$ \\
\hline
\end{tabular}

8 La plupart des manuels proposent également une ou deux doubles pages mettant en œuvre des activités plus ou moins alternatives, du type «Passé-Présent " (Nathan Cote), «Atelier de Clio » (LLS), «Regard critique " (Hatier) ou encore « Empreintes de l'Histoire » (Belin). Tous ont à cœur de faire alterner textes et images, en privilégiant ces dernières, souvent de grande taille, de proposer des cartes, des frises 
chronologiques ou encore des schémas de synthèse, et d'enrichir leurs contenus en renvoyant à des sites Internet, à des vidéos YouTube ou à d'autres types de ressources numériques. Tous enfin s'achèvent par deux ou trois doubles pages de révisions et de méthode, liées aux nouvelles épreuves du baccalauréat et qui sont généralement l'occasion d'introduire un ou deux documents supplémentaires. La quasi-totalité des manuels complètent le manuel "papier ", destiné avant tout aux élèves, par du contenu destiné aux professeur.es: ainsi Nathan a-t-il également publié une longue mise au point historiographique sur chaque chapitre, rédigée par des universitaires spécialistes de la question ${ }^{10}$ - en histoire médiévale, Emmanuelle Tixier du Mesnil. Ces productions seront exclues de l'analyse, pour se concentrer sur ce que les élèves peuvent voir et utiliser.

\section{Analyse des documents}

Venons-en, plus précisément, aux documents. Ceux-ci occupent l'essentiel de l'espace de ces chapitres: le cours ne représente en effet qu'une portion assez congrue du volume total. Le nombre de documents est assez variable d'un manuel à l'autre : avec 65 documents en tout, dont 33 documents iconographiques, Belin Colon propose presque deux fois plus de documents que Nathan Le Quintrec. Ce décalage est dû à la fois au nombre de pages différent - 34 pour Belin, 26 pour Nathan - et surtout à des maquettes différentes, qui permettent notamment à Belin de placer entre deux et quatre documents sur ses pages de cours (Tab. 3).

Tab. 3. Nombre et type de documents dans les différents manuels.

\begin{tabular}{|l|l|l|l|l|}
\hline & $\begin{array}{l}\text { Nombre de documents } \\
\text { iconographiques }\end{array}$ & $\begin{array}{l}\text { Nombre de } \\
\text { documents } \\
\text { textuels }\end{array}$ & $\begin{array}{l}\text { Nombre de documents } \\
\text { annexes (cartes, frises, } \\
\text { schémas, etc.) }\end{array}$ & Total \\
\hline Belin Colon & 33 & 20 & 12 & 65 \\
\hline Hachette & 22 & 15 & 12 & 48 \\
\hline Hatier Ivernel & 26 & 26 & 13 & 65 \\
\hline $\begin{array}{l}\text { LeLivreScolaire } \\
\text { Besson }\end{array}$ & 25 & 19 & 9 & 53 \\
\hline Magnard Vidal & 26 & 12 & 12 & 50 \\
\hline Nathan Cote & 14 & 13 & 12 & 34 \\
\hline $\begin{array}{l}\text { Nathan } \\
\text { Quintrec }\end{array}$ & 10 & 12 & 12 & 39 \\
\hline
\end{tabular}

10 L'origine de ces documents est bien plus intéressante à commenter. On repère aisément, à la lecture des différents manuels, des documents privilégiés, voire plébiscités. Ainsi de la description de Palerme par Ibn Jubayr ou du passage de Pierre le Vénérable dans lequel il explique son programme de traduction du Coran, deux textes 
utilisés dans six manuels. Le récit autobiographique d'Usama ibn Munqidh, émir syrien ayant vécu dans l'Orient des croisades, est mobilisé par cinq des sept manuels ${ }^{11}$, même si tous utilisent un extrait différent. De même le chrysobulle d'Alexis I $^{\text {er }}$ Comnène par lequel il donne d'importants privilèges commerciaux à Venise, daté de 1082, est-il utilisé par quatre manuels ${ }^{12}$.

11 Le tableau n'est guère plus varié pour les documents iconographiques. Il est ainsi tout à fait frappant que trois des sept manuels choisissent comme image d'ouverture du chapitre une enluminure ${ }^{13}$, certes différente, tirée des Cantigas de Santa Maria. On retrouve ici l'effet «banque d'images » évoqué plus haut, qui conduit à une relative uniformisation. La célèbre enluminure représentant une partie d'échecs entre un chrétien et un musulman, tirée du Libro de los juegos, est utilisée par quatre manuels ${ }^{14}$ : on mesure bien la profonde continuité qui existe entre ce chapitre et la question de médiévale inscrite à l'agrégation interne, dans la mesure où cette image faisait partie du sujet du concours lors de la session 2019.

La palme revient à la vue de Venise proposée par un manuscrit du Livre des Merveilles de Marco Polo conservé à la Bodleian Library ${ }^{15}$ : cette superbe enluminure est en effet utilisée par six des sept manuels, une quasi-unanimité à laquelle seul déroge Magnard encore n'y déroge-t-il pas totalement, car cette enluminure se retrouve sur sa couverture! Il s'agit de fait d'une image très célèbre, souvent utilisée pour la couverture d'ouvrages universitaires ou pour illustrer des articles de blog, l'une des premières à ressortir dès lors que l'on cherche des mots-clés comme "Venise Moyen Âge " sur un moteur de recherche; elle fait dès lors partie des "documents patrimoniaux » que tous les éditeurs vont avoir tendance à utiliser. Cette utilisation relève autant du choix - puisque l'un des enjeux du succès d'une collection est de ne pas "passer à côté » d'un document essentiel que les professeures s'attendent à retrouver dans le manuel - que de l'inconscient: ces images habitent les paysages mentaux des équipes éditoriales, qui vont logiquement avoir tendance à les utiliser. Il est intéressant de souligner combien l'image, si elle est ontologiquement identique, varie d'un manuel à l'autre, tant le cadrage choisi ou, surtout, les retouches photos peuvent la modifier. Elle baigne ainsi dans un nimbe rouge chez Belin, est fortement contrastée chez Hatier et semble au contraire très estompée chez LLS... Ces retouches ne modifient évidemment pas le contenu même de l'image, qui reste toujours lisible et donc offerte à l'analyse des élèves, mais invitent tout de même à se demander à partir de quel degré de modification une image devient autre.

Plusieurs cartes identiques apparaissent : ainsi d'une carte des États latins d'Orient ${ }^{16}$, de la péninsule Ibérique à l'époque de la Reconquista ${ }^{17}$, et surtout, point de passage oblige, du commerce vénitien, carte qui figure dans tous les manuels. Ces cartes se ressemblent d'autant plus que les manuels partagent tous un certain nombre de codes cartographiques, certains s'expliquant par des enjeux partagés de lisibilité, d'autres relevant là encore de l'inconscient collectif: ainsi le monde islamique est-il systématiquement représenté par un aplat vert. Enfin, certains personnages incontournables sont fréquemment présentés dans de petits encarts comme des "personnages repères »: c'est en particulier le cas de Saladin, qui apparaît dans six manuels ${ }^{18}$.

14 Cette impression de répétition est pourtant démentie par le nombre de documents, qui autorise chaque manuel à présenter un grand nombre de documents originaux. Si aucun manuel n'utilise donc que des documents uniques, aucun, à l'inverse, n'exploite 
seulement des documents que l'on trouverait également chez les autres. Au contraire même, car si l'on compte tous les documents, le bilan est sans appel : 156 documents n'apparaissent qu'une seule fois, 22 apparaissent deux fois, 25 trois fois et plus - dont 9 seulement cinq fois ou plus. La proportion de documents uniques oscille, par manuel, entre $48 \%$ et $70 \%$. C'est donc bien l'originalité qui domine, preuve de recherches documentaires approfondies de la part des équipes éditoriales. Certains documents pourtant très célèbres ne sont dès lors convoqués qu'une seule fois : ainsi du tympan de Conques (Hatier p. 67), de la mosquée-cathédrale de Cordoue (Belin p. 81) ou encore de l'Atlas Catalan (Nathan Le Quintrec p. 73). Bref, les manuels oscillent en permanence entre volonté de ne pas négliger un document célèbre - considéré comme incontournable et dont l'absence pourrait dès lors leur être reprochée - et effort pour se distinguer les uns des autres.

Même s'ils sont donc surtout différents, ces documents partagent un certain nombre de caractéristiques communes. D'abord, ils sont très majoritairement écrits par des hommes: seul LLS propose deux documents produits par des autrices, à savoir un extrait de l'Alexiade d'Anne Comnène et une enluminure dessinée par Jeanne de Montbaston. Deux documents sur un total d'un peu plus de deux cent : la proportion est donc infime, et recoupe le bilan très critique dressé, à l'échelle de l'ensemble des programmes d'histoire du secondaire, par Catherine Chadefaud ${ }^{19}$. Certes, l'immense majorité des documents médiévaux sont produits par des hommes, mais reste qu'on peut aisément trouver, à toutes les époques et dans des milieux sociaux plus variés qu'on ne l'a longtemps pensé, des autrices dont les textes pourraient être utilisés dans des manuels scolaires. Ce tableau est encore plus accablant quand on se tourne vers les biographies finales, deux ou trois doubles pages dans lesquelles les manuels proposent une compilation des portraits des "personnages clés » évoqués au fil des chapitres. Les femmes y brillent par leur absence, à la fois en général et pour le Moyen Âge en particulier : aucune femme médiévale n'est citée dans six des sept manuels. Seul LLS, à nouveau, en cite quatre - Aliénor d'Aquitaine, Christine de Pizan, Hildegarde de Bingen et Marie de France. Le bilan général n'est donc pas meilleur que celui fait par Eléna Suzat dans son mémoire sur le sujet, datant de $2009^{20}$. Il ne s'agit certes pas d'imposer des quotas, encore moins de chercher à tout prix une parité qui, pour l'époque médiévale, relève de l'impossible : reste néanmoins qu'il y a là un vrai enjeu, à la fois scientifique - car l'historiographie sur les places et les rôles des femmes au Moyen Âge est l'une des plus riches et des plus fécondes qui soit - et social. Comme le soulignent depuis plusieurs années des associations, les manuels scolaires peuvent être de formidables outils pour œuvrer à l'égalité des genres ${ }^{21}$ : encore faut-il, en l'absence de sollicitations venues des programmes eux-mêmes, que les équipes éditoriales relèvent le défi.

Un autre biais tient à l'origine géographique, ethnique et linguistique des auteur.es de documents. Les documents "occidentaux", autrement dit rédigés par des chrétiens obéissant au pape de Rome - des catholiques, pour faire simple, même si le terme est partiellement anachronique pour la période considérée - écrivant pour la majorité en latin et vivant en Occident, sont en effet largement majoritaires, composant au minimum $66 \%$ des documents (LLS) et au maximum $83 \%$ (Hatier). La moyenne tourne autour de $75 \%$. En outre, les rares documents non-occidentaux mobilisent souvent un petit nombre de sources: Usama ibn Munqidh, Ibn Jubayr, al-Sulami, une mosaïque byzantine, le chrysobulle d'Alexis Comnène. Même quand certains manuels font un effort pour présenter des textes issus d'auteurs arabes ou grecs, les juifs ou, plus 
encore, les chrétiens d'Orient brillent par leur absence, quasiment totale. Concernant les premiers, on trouve une poignée de documents traitant de leurs statuts légaux en Occident - législation d'Alphonse X, expulsion par un roi de France - ou en Orient statut des dhimmis ; mais il s'agit toujours de documents émis par des autorités nonjuives. Sur les sept manuels, on ne trouve que trois documents émis par un juif - LLS et deux fois Hatier -, et absolument aucun par un chrétien d'Orient. Il y a là un vrai enjeu d'équilibre : au-delà du profond biais européo-centriste que traduit cette répartition, comment veut-on espérer présenter une histoire équilibrée de la Méditerranée médiévale, une "histoire à parts égales ${ }^{22}$ ", en privilégiant à ce point les documents occidentaux ? Ce déséquilibre reproduit évidemment en partie un effet de sources bien connu - nous disposons de plus de textes pour l'Occident latin que pour l'Orient islamique -, lui-même dû à des conditions différentes d'élaboration et de conservation des écrits, et notamment des écrits documentaires, entre Orient et Occident ; mais il est aussi le fruit de la plus grande familiarité des équipes éditoriales avec l'histoire médiévale occidentale. Il contraste vivement avec l'actualité historiographique de ces domaines de recherche : les travaux de Camille Rouxpetel sur les chrétiens d'Orient ${ }^{23}$, par exemple, semblent totalement ignorés. Si l'absence d'un certain nombre de précisions et de nuances se comprend, encore une fois, par la nature même de l'objet étudié ici, reste que cette vision appauvrit singulièrement la richesse et la complexité de la Méditerranée médiévale. Quand Magnard écrit, dans ses révisions, que «la chrétienté est partagée en deux Églises par le schisme de 1054 ", non seulement il surestime l'importance de ce "schisme » qui en réalité n'en est pas un, comme les médiévistes le répètent depuis plusieurs années maintenant ${ }^{24}$, mais surtout il oublie totalement l'existence de ces Églises orientales qui, pendant des siècles et encore aujourd'hui, rassemblent plusieurs millions de fidèles à travers tout le bassin méditerranéen. De même quand Nathan Le Quintrec évoque l'établissement des États latins d'Orient en soulignant que «les populations musulmanes, juives et orthodoxes supportent mal [la] domination [des Francs] » (p. 78) : l'essentiel de la population de ces nouvelles structures politiques est en réalité des chrétiens d'Orient - Coptes, Jacobites, Maronites, Arméniens, etc. - qui s'accommodent fort bien de l'arrivée des Latins. Ce biais européo-centriste se donne également à voir à travers certaines formulations. Il semble par exemple étrange de parler de "reconquête » de Jérusalem par les croisés (Le Quintrec p. 78), dans la mesure où la Ville sainte n'a jamais été dominée par des catholiques, ou de dire que la victoire de Las Navas de las Tolosas permet à la Méditerranée de "redevenir" latine (Belin), un terme qui reproduit la vision d'une «reconquête» latine de la péninsule, schéma forgé par les souverains ibériques médiévaux afin de légitimer leurs entreprises militaires.

\section{Analyse des contenus}

Ce qui nous invite dans un deuxième et dernier temps à analyser davantage les contenus textuels, autrement dit les pages de cours, les révisions, les chapeaux introduisant les pages documents, etc. Le but n'est pas de faire une revue des erreurs, car aucun manuel - celui que j'ai dirigé moins que tout autre - ne peut prétendre être parfait. Tous doivent par définition faire des raccourcis, simplifier des concepts souvent extrêmement complexes, condenser plusieurs siècles d'histoire en quelques pages, le tout dans l'optique de préparer à des épreuves très formatées. Il s'agit donc 
moins de pointer du doigt des erreurs que de voir ce que ces erreurs disent quant à la connaissance et à la transmission de l'historiographie actuelle.

Les manuels, on l'a dit, sont tenus de respecter le programme officiel : on ne s'étonnera donc pas de trouver répété un peu partout le terme de "civilisation ", imposé par les textes alors même qu'il est depuis des années très critiqué par les historien-nes, qui dénoncent à la fois sa dimension essentialisante et son utilisation dans la théorie huntingtonienne du choc des civilisations ${ }^{25}$. De fait, le terme conduit à privilégier les continuités et tend à systématiquement rabattre l'identité d'une société donnée sur le fait religieux. Le manuel Hachette propose ainsi une double page introductive intitulée "unités et fractures entre les civilisations méditerranéennes", dans laquelle ces civilisations sont avant tout définies par leur religion respective: la civilisation chrétienne est ainsi illustrée par une mosaïque byzantine à la Vierge tandis que les civilisations musulmane et juive sont respectivement illustrées par la Kaaba et un rouleau de la Torah. Chez Nathan Cote, la civilisation occidentale est illustrée par une abbaye, la civilisation musulmane par une mosquée (p.64). Certes, l'hétérogénéité interne, notamment ethnique, de ces ensembles est bien rappelée dans l'ensemble de ces manuels : mais la religion reste présentée comme l'élément central, dans une vision finalement assez proche de celle avancée par Samuel Huntington. Certains manuels parviennent cependant à contourner subtilement les impératifs du programme: Magnard, notamment, consacre une double page à «la Méditerranée des monothéismes ", invitant à étudier les rapports entre les trois religions, sans jamais écrire, du moins dans cette double page, que ces dernières sont les fondements d'entités civilisationnelles. On se rapproche davantage ici d'une démarche historienne, ce qui permet également de rappeler aux élèves que les religions elles-mêmes ne sont pas figées dans le temps : loin d'être «à la racine » de quelconques civilisations, elles bourgeonnent en permanence et sont des faits sociaux qu'il s'agit d'étudier comme tels ${ }^{26}$.

19 On s'aperçoit également, sans surprise, que la plupart des manuels confondent occasionnellement ou systématiquement l'islam, comme religion, et l'Islam, comme culture et fait politique. De même, tous les manuels emploient-ils au moins une fois, et certains plusieurs dizaines de fois, le terme de "monde musulman", un terme profondément faussé car il occulte le fait que, jusqu'au XIII siècle au moins, la majorité de la population vivant dans le Dar al-islam n'est pas musulmane ${ }^{27}$. À nouveau, les juifs et les chrétiens d'Orient se voient comme effacés de l'histoire, relégués dans les limbes d'une non-existence. Plutôt que d'un "monde musulman", il convient, comme le rappellent régulièrement les spécialistes de la question, de parler de «monde islamique ", un terme qui permet de mettre l'accent sur le fait que c'est bien l'islam qui domine le paysage politique et juridique, tout en évitant de laisser croire que toute la population pratique la foi musulmane. Le terme même de «monde » devrait d'ailleurs être utilisé avec davantage de précautions. Il contribue puissamment à réifier les choses, laissant croire que ces sociétés voisines forment des entités parfaitement closes et étanches, quand l'examen de la réalité montre des situations bien plus poreuses ${ }^{28}$.

Les programmes, et donc les manuels, apparaissent ainsi en décalage flagrant avec l'historiographie actuelle qui, sans pour autant minimiser l'importance du fait religieux dans la construction tant du tissu social que des autorités politiques, montre avec finesse que les identités religieuses ne sont pas exclusives, et qu'elles s'articulent sans cesse à d'autres. Les travaux de Juliette Sibon sur les juifs de Marseille ${ }^{29}$, de Dominique 
Valérian sur les ports du Maghreb ${ }^{30}$, de Guillaume Saint-Guillain sur la Grèce latine ${ }^{31}$, parmi bien d'autres que l'on pourrait citer, ne semblent pas avoir été pris en compte. Même remarque quant à la notion de "tolérance ", très présente dans la plupart des manuels : au mieux nuancé d'un timide "une relative tolérance " (Magnard p. 59), le concept est le plus souvent utilisé tel quel, sans être discuté ou critiqué. On le retrouve dans les cours, dans les révisions ou même dans certaines activités : invitant l'élève à jouer le rôle d'un voyageur occidental décrivant la Tolède du XI siècle à son souverain, le manuel Hachette conseille "de montrer tout l'avantage de la tolérance religieuse " (p.93). Or, ce terme a été passé au crible de l'historiographie de ces vingt dernières années, qui ont montré combien ce concept, forgé à l'époque moderne, n'était pas à même de rendre compte de la complexité des situations médiévales ${ }^{32}$. Au-delà de l'anachronisme, il s'agit en fait d'un concept profondément opposé à la façon dont les sociétés médiévales pensent et vivent la diversité religieuse. Les travaux de David Nirenberg, en particulier, soulignent que ces sociétés multiculturelles et multireligieuses ne tendent pas vers une "tolérance» de l'autre, mais cherchent à construire un espace social partagé permettant une coprésence strictement régulée par la loi et, occasionnellement, par la violence ${ }^{33}$. On répondra, à raison, que ce dernier concept est particulièrement complexe et semble bien difficile à mobiliser devant des lycéen'nes : mais on peut au minimum éviter de répéter des erreurs - or l'idée d'une tolérance médiévale est une erreur -, et essayer d'introduire un peu de complexité - on pourrait par exemple attirer l'attention sur le fait que toutes les communautés ethniques et religieuses déploient leurs propres stratégies et ne cessent jamais d'être en compétition les unes avec les autres.

En règle générale, l'historiographie récente - et parfois pas si récente - semble mal connue ou, en tout cas, peu utilisée ${ }^{34}$. Il faudrait étudier plus précisément qu'on ne peut le faire ici les modalités de diffusion de cette historiographie récente, en essayant d'identifier les courroies de transmission qui fonctionnent le mieux - les revues L'Histoire et La documentation photographique semblent très utilisées - ou au contraire en relevant les médias les moins susceptibles d'être appropriés par les équipes éditoriales, comme les actes de colloque. Quand on se penche sur le détail des différents chapitres, on relève de nombreux éléments pertinents qui côtoient, sans hiérarchisation ni recul, des interprétations considérées actuellement comme largement dépassées. Ainsi, la croisade est-elle définie comme une entreprise servant à "canaliser la violence des seigneurs qui perturbe les royaumes d'Occident " (Nathan Le Quintrec p. 78), une vision que Jonathan Riley-Smith, entre autres, a pu battre en brèche ${ }^{35}$, et qu'on sait maintenant ne pas du tout correspondre à la réalité. Le manuel de Hachette explique quant à lui que les États latins ont été fondés pour " faire barrière à l'expansion arabe ", ce qui est doublement faux: d'une part parce que l'expansion musulmane est à ce moment-là portée par des Turcs bien plus que par des Arabes, la confusion entre les deux étant là aussi révélatrice de cette tendance à vouloir absolument penser le Dar alIslam comme un monde homogène; d'autre part et surtout parce que les croisades ne répondent pas à une quelconque "menace musulmane", car en 1095 les grandes invasions musulmanes sont terminées depuis longtemps. S'il s'agit en partie de répondre aux demandes d'aides de l'empereur byzantin, pressé par les Turcs - mais également par les Bulgares ou les Normands -, on sait désormais que les causes profondes de la première croisade sont bien davantage à chercher dans la vaste reconfiguration de la chrétienté occidentale qu'on appelle la réforme grégorienne : en appelant à délivrer les Lieux saints, le pape ne veut pas endiguer une quelconque 
invasion de païens, mais bien se poser comme le seul chef de file d'une chrétienté repensée comme une Église soudée et conquérante. Autre erreur, le « schisme de 1054 » est, presque partout, présenté comme une véritable rupture ${ }^{36}$, alors qu'on sait bien maintenant qu'il s'agit essentiellement d'un non-événement qui, s'il témoigne bel et bien d'une progressive dégradation des relations entre les chrétientés orthodoxe et catholique, n'est guère ressenti à l'époque comme une fracture ${ }^{37}$ : ce n'est qu'ensuite, et notamment après le sac de Constantinople en 1204, que cette dispute théologique est redécouverte et réinventée pour devenir l'une des étapes clés sur la route qui mène à la séparation entre catholiques et orthodoxes. Autre erreur, ou en tout cas autre simplification qui pourrait être évitée, la plupart des manuels diffusent une vision assez binaire, opposant des contacts «conflictuels» et des contacts apaisés, à la fois commerciaux et culturels. La vision tombe parfois dans des formules assez manichéennes du type « le commerce méditerranéen contribue à pacifier les relations entre chrétiens et musulmans » (Nathan Le Quintrec p. 85). Claude Denjean a pourtant bien montré qu'il était impossible d'opposer ainsi commerce et conflit : non seulement les deux s'enchevêtrent en permanence, mais surtout l'existence de relations commerciales, y compris quand elles s'inscrivent dans la durée, ne conduit en rien à l'établissement de rapports sociaux plus apaisés ${ }^{38}$. Signalons enfin que pour ce qui est des sources textuelles, les éditions scientifiques récentes sont généralement peu utilisées : les manuels préfèrent renvoyer à des compilations de sources, parfois datées, ou à des traductions du XIX ${ }^{e}$ siècle librement accessibles sur Internet ${ }^{39}$.

Il serait injuste de clore cet article sur cette critique. De très nombreux éléments sont au contraire bien présentés. Tous les manuels consacrent ainsi plusieurs lignes, voire plusieurs pages, aux transferts culturels entre l'Orient et l'Occident, en mentionnant les transferts de savoirs médicaux ou philosophiques, les échanges technologiques, les brassages d'idées et de produits - ici le sucre, là les chiffres, ailleurs la médecine... Certes, on pourrait légitimement critiquer le fait que ces apports s'ancrent toujours autour des mêmes exemples: Tolède, Cordoue, Palerme trônent sur le podium et semblent, quand on sait que c'était déjà le cas en 1995, indétrônables. À quand des études sur Antioche, sur Chypre, sur Malte ou sur Montpellier, autant de lieux qui ont été eux aussi au premier plan des échanges commerciaux et culturels? Reste, malgré ces répétitions, que les chapitres multiplient les approches intelligentes et originales. Nathan Le Quintrec rapproche ainsi la Méditerranée médiévale et contemporaine pour rappeler que les flux de migrants font partie de son histoire; Belin propose d'étudier « l'empreinte de l'histoire » à travers la patrimonialisation touristique du centre urbain de Cordoue. Les manuels ont à cœur de convoquer des textes d'historiens - Vincent Lemire, Henri Bresc et Geneviève Bresc-Bautier, Alain Ducellier, Alain Demurger -, de présenter une grande variété de sources, d'enrichir leurs chapitres en renvoyant à des œuvres de fiction contemporaines - Le Destin de Youssef Chahine, Kingdom of Heaven de Ridley Scott, le superbe Stupor Mundi de Néjib, etc. Ce faisant, par cette diversité documentaire, les sept manuels scolaires savent également, à leur échelle et malgré des contraintes particulièrement fortes, rendre justice à la complexité de l'histoire médiévale. 


\section{Conclusion}

En creux, ce tableau trop rapide interroge les médiévistes sur leur capacité individuelle et collective - à diffuser leurs recherches. Reste en effet à la lecture de ces chapitres une impression diffuse d'occasion manquée: l'un dans l'autre, et sauf exception ponctuelle, force est de constater que les manuels scolaires de 2019 n'apportent guère plus que leurs ancêtres de 1995-1996. On y trouvait déjà les mêmes pages sur les transferts culturels, les mêmes dossiers consacrés au trio Palerme/ Cordoue/Tolède, le même souci d'équilibre, parfois jusqu'au manichéisme, entre contacts " conflictuels » et contacts " pacifiques». Pour un médiéviste, a fortiori pour un spécialiste de la Méditerranée médiévale, cette non-évolution des manuels scolaires, en vingt-cinq ans, est aussi frappante que préoccupante : le champ historiographique a en effet été entre-temps profondément renouvelé par une série de travaux majeurs. Il serait faux, bien sûr, de dire que rien n'a changé dans les manuels scolaires de classe de seconde, et l'on détecte heureusement de nombreux éléments reflétant des renouvellements historiographiques. Ainsi, par rapport à ceux de 1995, aucun manuel contemporain ne présente-t-il plus al-Andalus sous le prisme de la convivencia : ce vieux fantôme historiographique, bien dénoncé entre autres par Christophe Cailleaux, semble - définitivement? - exorcisée ${ }^{40}$. De même, l'insistance de tous les manuels à rappeler "l'avance intellectuelle» dont jouit le monde islamique au début de la période et à mentionner les transferts de savoir entre Orient et Occident s'explique-t-elle probablement par l'effet des nombreuses réponses, souvent bien médiatisées, à "l'affaire Gouguenheim " ${ }^{41}$. Mais ces éléments sont une exception : le plus souvent, les manuels du secondaire continuent à présenter l'histoire de la Méditerranée médiévale sur le même mode, avec les mêmes exemples et à partir des mêmes sources qu'il y a une génération. S'il est normal qu'il y ait un décalage temporel entre le moment où une recherche historique est produite et le moment où elle est suffisamment établie, acceptée et connue pour pouvoir être diffusée auprès de lycéen-nes, vingt-cinq ans semblent un délai bien long pour un tel processus.

Le programme a évidemment sa part, majeure, de responsabilité : difficile par exemple de proposer un chapitre équilibré quant à l'origine des sources lorsque les deux «Points de passage et d'ouverture » imposés portent tous deux sur l'Occident... De même, on peut penser que les délais très courts entre la date de sortie du programme et son entrée en vigueur empêchent les équipes éditoriales d'innover, en les privant du temps de lire, d'échanger avec des universitaires, de découvrir de nouveaux sujets.

Mais le programme n'est pas le seul responsable. Les renouvellements contemporains de la médiévistique, faits de recherches en cours, de nouveaux terrains, de nouvelles méthodes, d'éditions scientifiques récentes, sont encore trop peu connus des équipes éditoriales. Dès lors, les manuels ne peuvent guère espérer présenter ces renouvellements aux professeures, qui, à leur tour, auront forcément plus de mal à les introduire à leurs élèves. Les manuels pourraient être au contraire une superbe chambre de résonance, un relais pour toucher des milliers de lycéen.nes, pour leur montrer du même coup la richesse de l'histoire médiévale et le dynamisme de la médiévistique contemporaine. En plaçant au centre du chapitre des concepts qui sont parmi les plus travaillés par les médiévistes ces dernières années - religion, violence, tolérance, minorités, transferts culturels, commerce -, et qui ne sont pas en outre dénués d'une certaine actualité politique et sociale, le programme devrait permettre 
aux manuels d'aborder quelques-uns des apports récents de la recherche, voire d'évoquer, fût-ce en les simplifiant, certains des débats contemporains. En plus de l'enjeu proprement scientifique, consistant à vouloir élaborer des manuels scolaires aussi à jour que possible, il est permis de penser qu'il y a là un enjeu social assez brûlant : à l'heure où les réformes récentes, en cours ou à venir, mettent à mal les conditions de production de la science historique en fragilisant plus encore l'institution universitaire, il y a tout à gagner à construire des manuels scolaires à même de rappeler que l'histoire est avant tout une science, qui évolue avec la société dans laquelle elle se fait et qui nécessite pour être exercée la présence d'historien-nes professionnelles.

26 Ces derniers ont de fait un rôle important à jouer dans la construction de ce médiévalisme scolaire. En amont, ils et elles peuvent chercher à intervenir dans la rédaction des programmes, même si les institutions savantes, consultées par le ministère, dénoncent souvent avec amertume le peu de cas qui est fait de leur avis ${ }^{42}$. Les médiévistes gagneraient également à s'investir concrètement dans la rédaction des manuels scolaires du secondaire : la quasi-totalité des éditeurs font en effet appel à des " comités scientifiques » composés d'universitaires, chargés de relire les chapitres pour les corriger. Le nombre important de refus que, dans mes fonctions de directeur d'ouvrage, j'ai essuyé en proposant à des universitaires de jouer ce rôle montre qu'il s'agit là d'une fonction dont l'intérêt ou la légitimité sont peu reconnus. Les manuels scolaires, aussi raccourcis et donc frustrants soient-ils, constituent pourtant un superbe moyen de toucher pendant des années un public très large, en même temps qu'un terrain concret où peut se nouer un lien entre secondaire et supérieur. Plus généralement encore, les manuels scolaires construisent - ou plutôt contribuent à construire - ce qu'on peut désigner comme un véritable médiévalisme scolaire, à savoir une vision du Moyen Âge construite à la fois par et pour l'école, qui touche chaque année un énorme public. Ce médiévalisme scolaire reste globalement peu analysé : il serait pourtant très intéressant d'étudier les manuels scolaires du collège ou de l'école primaire, mais également toute la littérature parascolaire - les cahiers de vacances, par exemple - ou encore les différentes ressources pédagogiques proposées sur le site du ministère ou des différentes académies, afin d'analyser précisément la vision de la période médiévale, en en soulignant notamment les évolutions, ou, en l'occurrence, les non-évolutions. Il y aurait beaucoup à tirer de telles analyses, qui permettraient de s'interroger concrètement sur la manière dont les nouvelles connaissances, hypothèses et recherches historiographiques se diffusent dans la société.

Enfin et surtout, le tableau assez négatif dépeint dans cet article invite en creux les médiévistes à réfléchir plus que jamais à la question de la diffusion de leurs recherches. Le bouillonnement actuel d'initiatives allant dans ce sens, qui voit des chercheures s'investir dans des podcasts, des conférences grand public ou encore dans la rédaction de bandes dessinées, est à cet égard porteur d'espoir : on peut penser que de tels media sauront, mieux ou en tout cas plus rapidement que des revues savantes ou des ouvrages publiés par des éditions universitaires, diffuser auprès des professeures et des maisons d'édition l'actualité historiographique. Les cafés virtuels organisés par l'Association des professeurs d'histoire et de géographie (APHG) apparaissent également comme une excellente initiative, une manière de présenter aux professeures l'actualité de la recherche sur des thèmes variés, tout en laissant la parole aux professionnelles de l'histoire : on pourrait tout à fait imaginer que les futurs manuels se nourrissent de ces conférences, soit pour proposer des dossiers originaux, soit pour faire travailler les 
élèves sur ces formats. De même, la parution à l'automne 2020 d'un manuel scolaire de Licence rédigé collectivement par des universitaires et disponible gratuitement sur Internet traduit une prise de conscience collective de l'enjeu crucial que représentent les supports scolaires et parascolaires pour la diffusion des connaissances historiques ${ }^{43}$. À quand, donc, un manuel du secondaire rédigé, sinon par, du moins avec des universitaires, qui ne renoncerait ni à l'impératif pédagogique de simplification ni à l'ambition de s'inscrire à la pointe de l'historiographie récente, et qui saurait ainsi renouveler en profondeur ce médiévalisme scolaire?

Reçu : 23 juin 2021 - Accepté : 5 décembre 2021

\section{NOTES}

1. Voir notamment L. DE COCK, «L'enseignement de l'histoire est pris en étau », Ballast, 23 avril 2019, en ligne [https://www.revue-ballast.fr/laurence-de-cock-lenseignement/], consulté le 6 décembre 2021.

2. Sans être exhaustif, on peut ainsi citer le travail de Manon Bril (C'est une autre histoire), d'Ugo Bimar (Confessions d'Histoire), de Benjamin Brillaud (Nota Bene) ou encore de Laurent Turcot (L'Histoire nous le dira).

3. Voir V. chambarlhac, «D'où viennent les savoirs des élèves? ", in F. LANTHEAume et J. LÉTOURNEAU (dir.), Le récit du commun. L'histoire nationale racontée par les élèves, Lyon, 2016, p. 39-47.

4. Sur la manière dont le collège et le lycée traitent la période médiévale, on lira avec intérêt deux articles: S. Abiker, «Les lettres médiévales à l'école élémentaire. État des lieux et propositions. ", Perspectives médiévales, 36, 2015, en ligne [http://journals.openedition.org/ peme/7342], consulté le 13 janvier 2022, et P. Prétou, «La violence seigneuriale à la fin du Moyen Âge, vue par les représentations pédagogiques en France ", Criminocorpus, en ligne [http:// journals.openedition.org/criminocorpus/9388], consulté le 13 janvier 2022.

5. Accessible en ligne [ https://www.lib-manuels.fr/textbook/5d264285451c9549068745ac? demo=true\&page=283], consulté le 6 décembre 2021 .

6. Accessible en ligne [https://fr.calameo.com/read/004822953bfbc87aa6fac], consulté le 6 décembre 2021.

7. Accessible en ligne [ https://fr.calameo.com/read/0005967292db34c17fc6e], consulté le 6 décembre 2021.

8. Pour donner une idée de cette contrainte de place : dans le manuel du LivreScolaire.fr que j'ai dirigé, les cours occupent quatre pages simples et représentent en tout, titres et problématiques comprises, un peu moins de 15000 signes (espaces comprises). S'y ajoutent à l'échelle du chapitre environ 6000 signes de texte (texte d'ouverture du chapitre, chapeaux introduisant les doubles pages documentaires, page méthode, etc.). En tout, l'auteur·e dispose donc de moins de 25000 signes pour traiter de l'ensemble du chapitre. Tous ceux qui se sont déjà prêtés à l'exercice pénible de réduire leurs textes pour rentrer dans un moule aussi strict - et encore, même les actes de colloque les moins généreux accordent généralement 30000 signes - comprendront facilement combien cette contrainte pèse sur la rédaction de manuels scolaires. 
9. Voir M.-C. BAQUÈs, «L'évolution des manuels d'histoire du lycée. Des années 1960 aux manuels actuels », Histoire de l'éducation, 114 (2007), en ligne [https://journals.openedition.org/histoireeducation/1249], consulté le 6 décembre 2021.

10. S. COTE et E. PICARD, Regards historiques sur les grandes étapes de la formation du monde moderne, Paris, 2019.

11. Belin, Hachette, Hatier, LLS et Magnard.

12. Belin, Hatier, Nathan Cote et Nathan Le Quintrec.

13. Belin, Hatier et LLS.

14. Belin p. 85, Hachette p. 74, Hatier p. 82 et Magnard p. 68.

15. Le manuscrit MS 264, fol. 218r, accessible en ligne [https://iiif.bodleian.ox.ac.uk/iiif/viewer/ d21e23ac-11a8-4d24-ad4d-07ad10e0ea34/\#?

$\mathrm{c}=0 \& \mathrm{~m}=0 \& \mathrm{~s}=0 \& \mathrm{cv}=456 \& \mathrm{r}=0 \& \mathrm{xywh}=-3171 \% 2 \mathrm{C}-380 \% 2 \mathrm{C} 11680 \% 2 \mathrm{C} 7574]$, consulté le 6 décembre 2021.

16. Belin, Hachette, Hatier, Nathan Cote, Nathan Le Quintrec et Magnard.

17. Belin, Hachette, Hatier, Nathan Cote, Nathan Le Quintrec et Magnard.

18. Belin, Hachette, Nathan Cote, Nathan Le Quintrec, LLS et Magnard.

19. C. CHADEFAUD «Les femmes toujours absentes de la société, de l'économie et des civilisations. Un constat accablant dans les nouveaux programmes d'histoire de lycée ", Historiens et géographes, 448 (décembre 2019), p. 15-24.

20. E. SUZAT, «Les représentations du genre dans les manuels d'histoire du lycée, 1968-2008 ", mémoire soutenu sous la direction de G. Vergnon, IEP de Lyon, 2009, accessible en ligne [http:// doc.sciencespo-lyon.fr/Ressources/Documents/Etudiants/Memoires/Cyberdocs/MFE2009/ suzat_e/pdf/suzat_e.pdf], consulté le 21 janvier 2021.

21. Voir notamment le guide édité par le centre Hubertine Auclert, «Faire des manuels scolaires des outils de l'égalité entre les femmes et les hommes ", 2014, accessible en ligne [https:// www.centre-hubertine-auclert.fr/sites/default/files/fichiers/cha-guide-manuels-scolaires-2019web.pdf], consulté le 21 janvier 2021. Voir également l'épisode 4 du podcast réalisé par l'association Mnémosyne, «Le genre des manuels scolaires", accessible en ligne [https:// www.mnemosyne.asso.fr/mnemosyne/podcast/04-le-genre-des-manuels-dhistoire/], consulté le 6 décembre 2021.

22. Selon l'expression de R. BERTRAND, L'Histoire à parts égales : récits d'une rencontre Orient-Occident, $X V I^{e}-X V I I^{e}$ siècle, Paris, 2014.

23. C. ROUXPETEL, L'Occident au miroir de l'Orient chrétien: Cilicie, Syrie, Palestine et Égypte (XIIXIV siècle), Rome, 2015.

24. Parmi de nombreux travaux possibles, citons uniquement l'article de J.-C. CHEYNET, « Le schisme de 1054 : un non-événement? ", in C. CARozzi et H. TAVIANI-CAROZZI (dir.), Faire l'événement au Moyen Âge, Aix-en-Provence, 2007, p. 299-312.

25. B. DUFAL, «Faire et défaire les civilisations ", in P. BÜTTGEN et alii (éd.), Les Grecs, les Arabes et nous. Enquête sur l'islamophobie savante, Paris, 2009, p. 317-358.

26. Voir A. NEF, L'Islam a-t-il une histoire ? Du fait religieux comme fait social, Lormont, 2017.

27. Voir entre autres : R. ELLENBLUM, «Demography, Geography and the Accelerated Islamisation of the Eastern Mediterranean », in I. KATZNESLON et M. RUBIN (dir.), Religious Conversion: History, Experience and Meaning, Burlington, 2014, p.61-80; P. WOOD, "Christians in the Middle East, 600-1000 : Conquest, Competition and Conversion ", in A. PEACOCK, B. DE NICOLA et S. NUR YILDIZ (dir.), Islam and Christianity in medieval Anatolia, Burlington, 2015, p. 24-50.

28. Je me permets de renvoyer à $F$. BESSON, «Vivre en communauté ou entre communautés? Une réflexion sur le middle ground des États latins d'Orient », Questes, 32 (2016), p. 35-50.

29. J. SIBON, Les Juifs de Marseille au XIV siècle, Paris, 2011. 
30. D. VALÉRIAN, «Marchands latins et sociétés portuaires dans le Maghreb médiéval. Le rôle central des intermédiaires ", in C. QUERTIER, R. CHILÀ et N. PLUCHOT (dir.), "Arriver » en ville. Les migrants en milieu urbain au Moyen Âge, Paris, 2013, p. 213-223.

31. G. SAINT-GUILLAIN et D. STATHAKOPOULOS, Liquid \& Multiple: Individuals \& Identities in the Thirteenthcentury Aegean, Paris, 2012.

32. Voir par exemple la très efficace mise au point de Z. BOU AKL, «La tolérance d'Averroès ? Les pièges d'une approche anhistorique ", in V. SALES et N. WEILL-PAROT (dir.), Le vrai visage du Moyen Âge. Au-delà des idées reçues, Paris, 2017, p. 215-228.

33. Voir notamment D. NIRENBERG, Violence et minorités, Paris, 2001 et plus récemment D. NIRENBERG, Neighboring faiths : Christianity, Islam, and Judaism in the Middle Ages and today, Chicago, 2016.

34. Redisons une fois de plus qu'il ne s'agit en aucun cas de blâmer les équipes éditoriales. Pour le chercheur professionnel, la critique est toujours facile, car tout semblera toujours raccourci. Dès lors que l'on accepte de prendre en compte les multiples contraintes qui pèsent sur la production de manuels scolaires, c'est au contraire une grande admiration que l'on ressent face à la capacité de synthèse et à l'intelligence pédagogique qui éclatent dans chaque page de chaque manuel.

35. Voir par exemple J. RILEY-SMITH, "Crusading as an Act of Love », History, 65-214 (1980), p. $177-192$.

36. Par exemple Hatier p. 73 : «en 1054, c'est le schisme. L'Église orthodoxe du patriarche se sépare de l'Église catholique du pape».

37. Voir notamment C. GASTGEBER, "The So-Called Schism of 1054 and its Impact on Byzantine Society ", in B. CROSTINI et G. PEERS (dir.), A Book of Psalms from Eleventh-Century Byzantium, Cité du Vatican, 2016, p. 193-227.

38. C. DENJEAN, « Les Juifs courtiers parmi les chrétiens : l'échange sans la religion? », in Cahiers de recherches médiévales et humanistes, 24 (2012), p.197-212, disponible en ligne [https:// journals.openedition.org/crm/12919], consulté le 6 décembre 2021.

39. Il est permis de penser, quand on regarde de près les textes utilisés, que le site de Philippe Remacle joue un rôle essentiel en mettant à disposition de nombreuses éditions du XIX ${ }^{e}$ siècle. Mais ces traductions sont souvent partielles et datées, ce qui donne des textes, dans les manuels scolaires, parfois bien éloignés de leur version originale - au point, dans certains cas extrêmes, de verser dans de la pure et simple écriture d'invention. Je développerai cet aspect dans un futur article.

40. C. Cailleaux, «Chrétiens, juifs et musulmans dans l'Espagne médiévale. La convivencia et autres mythes historiographiques ", Cahiers de la Méditerranée, 86 (2013), p. 257-271; disponible en ligne [https://journals.openedition.org/cdlm/6878], consulté le 6 décembre 2021.

41. Voir P. BÜTTGEN, A. DE LIBERA, M. RASHED et I. ROSIER-CATACH (dir.), Les Grecs, les Arabes et nous. Enquête sur l'islamophobie savante, Paris, 2009.

42. Voir P. LEGRIS, Qui écrit les programmes d'histoire ?, Grenoble, 2014.

43. R.-M. BÉRARD, B. GIRAULT et C. KIKUCHI (dir.), Initiation aux études historiques, Paris, 2020.

\section{RÉSUMÉS}

Les nouveaux programmes d'histoire du secondaire, entrés en vigueur en septembre 2019, imposent en classe de seconde générale un chapitre consacré à l'étude de la Méditerranée médiévale. Comme à chaque réforme des programmes, les éditeurs de manuels scolaires ont fait 
paraître de nouveaux manuels, publiés à la rentrée 2019. Cet article analyse la manière dont sept manuels traitent de la période médiévale, en s'intéressant en particulier, via une analyse à la fois quantitative et qualitative, aux documents textuels et iconographiques choisis, à l'historiographie mobilisée, aux exemples mis en avant. Supports de diffusion des connaissances, les manuels scolaires sont également l'un des lieux principaux où se construit un médiévalisme scolaire, autrement dit une certaine représentation de la période médiévale diffusée à un public de lycéens et lycéennes, sur laquelle il convient de réfléchir en profondeur.

The new high school history programs, which came into effect in September 2019, require a chapter devoted to the study of the medieval Mediterranean in the tenth grade. As with every curriculum reform, school textbook publishers have published new textbooks. This article analyzes the representations of the medieval period in these seven textbooks, via an analysis quantitative and qualitative. Textbooks can be considered as one way to built and to diffuse a school medievalism, in other words a certain representation of the medieval period transmited to a public of high school student.

\section{INDEX}

Mots-clés : médiévalisme, manuels scolaires, éducation, diffusion de la recherche, historiographie, programmes scolaires

Keywords : medievalism, school textbooks, education, dissemination of research, historiography, school curricula

\section{AUTEUR}

\section{FLORIAN BESSON}

Docteur en histoire médiévale de l'université Paris-Sorbonne 